\title{
Data-Driven Control Based on the Interval Type-2 Intuition Fuzzy Brain Emotional Learning Network for the Multiple Degree-of-Freedom Rehabilitation Robot
}

\author{
Hongmei Li, ${ }^{1}$ Di Li, ${ }^{2}$ Xiangjian Chen ${ }^{D}{ }^{1},{ }^{1}$ and Zhongqiang Pan ${ }^{1}$ \\ ${ }^{1}$ Jiangsu University of Science and Technology, Zhenjiang, China \\ ${ }^{2}$ China Shipbuilding Industry Corporation 723, Yangzhou, China \\ Correspondence should be addressed to Xiangjian Chen; jsjxy_cxj@just.edu.cn
}

Received 2 September 2020; Revised 9 November 2020; Accepted 28 November 2020; Published 16 January 2021

Academic Editor: Luigi Rodino

Copyright (c) 2021 Hongmei Li et al. This is an open access article distributed under the Creative Commons Attribution License, which permits unrestricted use, distribution, and reproduction in any medium, provided the original work is properly cited.

\begin{abstract}
A novel interval type-2 intuition fuzzy brain emotional learning network model (IT2IFBELC) which depends only on the input and output data is proposed for the rehabilitation robot, which is different from model-based control algorithms that require exact dynamic model knowledge of the rehabilitation robot. The proposed model takes advantage of the type- 2 intuition fuzzy theory and brain emotional neural network, and this is no rule initially; then, the structure and parameters of IT2IFBELC are tuned online simultaneously by the gradient approach and Lyapunov function. The system input data streams are directly imported into the neural network through an interval type-2 intuition fuzzy inference system (IT2IFIS), and then the results are subsequently piped into sensory and emotional channels which jointly produce the final outputs of the network. That is, the whole controller is composed of three parts, including the ideal sliding mode controller, the interval type-2 intuition fuzzy brain emotional learning network controller, and a powerful robust compensation controller, and then one Lyapunov function is designed to guarantee the rapid convergence of the control systems. For further illustrating the superiority of this model, several models are studied here for comparison, and the results show that the interval type- 2 intuition fuzzy brain emotional learning network model can obtain better satisfactory control performance and be suitable to deal with the influence of the uncertainty of the rehabilitation robot.
\end{abstract}

\section{Introduction}

Rehabilitation robots can significantly improve the motor ability and quality of life of people with reduced limb function. With the acceleration of the aging process, the number of patients with stroke, Parkinson's disease, spinal injury, and lower extremity paraplegia will continue to increase. Even after professional rehabilitation or treatment, the body functions of this group of people can be restored to a certain degree, but it is difficult to return to their original state. Most people's physical control ability becomes lower, and walking will become a very difficult task. So, their daily lives will be affected seriously by the activities and qualities. From the point of view of population aging trend and the growth of the potential beneficiary population, the study of the full-body assisted rehabilitation robot for the elderly and disabled can not only improve the motor ability, but also promote the social harmonious development. However, existing rehabilitation robots are generally larger in size and heavier in weight. Adaptation to the ground and flexibility of movement still need to be further improved. The working mode of most rehabilitation systems is that the wearer is carried by the robot, and the gait of different users is not fully coordinated. Although there are several products that can meet the requirements of rehabilitation robot control, the coupling performance of the human rehabilitation robot used to help the elderly and disabled is still very insufficient. One therapist rehabilitation robot in this paper is designed to be worn to provide rehabilitation therapy for the stroke patients [1-4]. The effective control strategies are so important for the rehabilitation robot to operate coordinately with the human upper limb. 
To help the stroke patients, one therapist rehabilitation robot was designed in this paper [1-4]; this robot is so complicated to be operated in control to work with the human upper limb, and the general control methods $[5,6]$ could be used for this robot. Liu et al. [7] proposed the adaptive fuzzy output-feedback tracking control for switched stochastic pure-feedback nonlinear systems; the above model-based methods could be used for the robot control, and the other methods which used the gravity and friction compensation controller also gained effective control performances [8-10]. Liu et al. [11-14] proposed the fuzzy model, neural network backstepping, and adaptive fuzzy model control of fractional-order delayed systems. Recently, researchers began to use more advanced control methods. However, most of the control methods are designed based on the model of the robot. Since the interacting model of this robot is time varying with uncertainty, the controller based on the model scheme is not very effective. With the development of the technology, the data-driven method now is an alternative effective way, which does not need the model of the interacting model of the rehabilitation robot, and the information can be obtained directly from the recorded process [15].

Data-driven control (DDC) method does not contain mathematical model information of the controlled process, which only uses online or offline process data to design the controller. DDC has characteristics of convergence, stability, and robustness under certain assumed conditions. In [16], the authors stated the necessity of the data-driven control theory in detail from three aspects, which contain the control theory, the application of control theory, and the development of control theory. Data-driven control technology is widely used in transportation [17], machinery manufacturing [18], chemical production [19], and motor control [20].

The sliding mode control (SMC) is one of the effective methods to handle the nonlinear systems with uncertainty by using the model-based or data-based control scheme; for example, in [21], the authors proposed a model-free adaptive sliding mode robust control with neural network estimator for the robot, which combined the sliding model control and the neural network into the model-free method. However, some researchers have proved that the control input data stream has been chattered by a combination of uncertainties through different kinds of pathways. To solve this problem, some studies have shown collaborating with the artificial neural network to enhance the control performance under uncertainty. The concept of brain emotional learning (BEL) networks was defined by LeDoux in 1991 [22-24]. Compared to other neural networks, the BEL network could achieve faster learning speed and better approximation ability for its special structure, which means that the BEL has two parts including an orbitofrontal cortex and an amygdala that are responsible for human emotional sensing and analyzing. Thus, in recent years, the BEL has been used for various fields such as prediction, identification, and control [25-33]. Meanwhile, the fuzzy neural network (FNN) takes advantages of both fuzzy logic and neural networks. FNN could not only process quick learning, but also offer one unique and flexible framework of knowledge representation.
So, the combination of the fuzzy logic set with the brain emotional learning network was presented [34], in which one self-organizing emotional learning network was constructed for the intelligent robot control. And, in reference [35], the fuzzy emotional learning network combined with the sliding model control method was proposed for the nonlinear system; meanwhile, this method was also implemented in humanoid robots [35]. In reference [36], the authors introduced the wavelet function to the fuzzy emotional learning network combined with the sliding model control method for the MIMO nonlinear system. These fuzzy logic sets used in the above references are all based on type-1; however, the type- 2 fuzzy sets could handle the uncertainties more flexibly for more adjustable parameters contained in the structure, which could decrease the difficulty in uncertainty representation compared to the type- 1 fuzzy sets. Therefore, the authors have proposed one hybrid controller for the robots and chaotic system, and the hybrid controller was composed of sliding model surface, type-2 fuzzy sets, brain emotional learning network, and one robust compensation [37].

Recently, an interval type-2 intuition fuzzy system (IT2IFS) was introduced by Chao et al. [38], where an intuition fuzzy index and nonmembership functions are imported into the interval type- 2 fuzzy logic system. The interval type- 2 intuition fuzzy system enhances the ability to deal with uncertainties by using the IF index in the FOUs of the MFs and NMFs through a process of scaling and shifting. The IT2IFS with the Takagi-Sugeno-Kang type (IT2IFLS-TSK) was proposed in reference [39], where gradient descent and decoupled extended Kalman filter were adopted to handle the structure and parameter learning. IT2IFLS was also implemented in time series and nonlinear system prediction $[11,40]$. Le et al. [41] present a design of interval type-2 fuzzy brain emotional learning control combining with the selfevolving algorithm to help the network to automatically achieve the optimum construction.

Motivated by the above discussions, this paper proposes one novel interval type-2 intuition brain emotional learning network controller and uses it in the multiple degree-offreedom rehabilitation robot control. The main contributions of this paper include the following: (1) the novel interval type- 2 intuition brain emotional learning network model is proposed firstly; (2) the parameters can be tuned online by adaptive laws; (3) the structure of the interval type-2 intuition brain emotional learning network can be constructed automatically from the empty initial rule; (4) the stability of this proposed control system is guaranteed by Lyapunov function; and (5) numerical simulations have been made to demonstrate the effectiveness of the proposed method for the multiple degree-of-freedom rehabilitation robot. This paper is organized as follows: Section 2 introduces the concept of the interval type-2 intuition fuzzy set and the brain emotional learning network. Section 3 presents the structure of the novel interval type- 2 intuition brain emotional learning network model, the parameters learning, and the self-organizing structure learning algorithms. Section 4 describes the overall control framework for the multiple degree-of-freedom rehabilitation robot by 
combining the sliding surface, the interval type-2 intuition brain emotional learning network controller, and one robust compensation, and also the stability of this proposed scheme has been provided. Section 5 shows the simulation results. And finally, the conclusions are given in Section 6.

\section{Overview of Fundamental Concepts}

In this section, we present the preliminary concepts of the type-2 intuition fuzzy set theory and brain emotional network.

\subsection{Interval Type-2 Intuition Fuzzy Set}

Definition 1. A generalized type-2 intuition fuzzy set is composed of type-2 membership function $\mu_{A}^{* *}(x, u)$ and a type-2 nonmembership function $v_{\widetilde{A}^{*}}(x, u)$ :

$$
\widetilde{A}^{*}=\left\{\begin{array}{c}
(x, u), \mu_{A}^{*}(x, u), \nu_{A}^{*}(x, u) \\
\mid \forall u \in J_{x}^{u}, \forall v \in J_{x}^{v}
\end{array}\right\} .
$$

Therefore, we get

$$
\begin{aligned}
0 & \leq \mu_{A}^{* *}(x, u), \\
\mathcal{\nu}_{A}^{* *}(x, u) & \leq 1,
\end{aligned}
$$

where $\forall u \in J_{x}^{u}, \forall v \in J_{x}^{v}$

$$
\begin{aligned}
& J_{x}^{u}=\left\{(x, u): u \in\left[\underline{u}_{A}^{*}(x), \bar{u}_{\widetilde{A}^{*}}(x)\right]\right\}, \\
& J_{x}^{v}=\left\{(x, v): v \in\left[\underline{v}_{A}^{*}(x), \bar{v} \widetilde{A}^{*}(x)\right]\right\},
\end{aligned}
$$

and a T2IFS can be expressed as

$$
\int_{x \in X} \frac{\left[\int_{u \in J_{x}^{u}} \int_{v \in J_{x}^{v}}\left\{\mu \widetilde{\sim}_{A}^{*}(x, u), \nu_{A}(x, u)\right\}\right]}{(x, u)} .
$$

When $v_{\widetilde{A}^{*}}(x, u)=1$ and $\mu_{A}^{* *}(x, u)=1$, the type-2 intuition fuzzy set will become interval type- 2 intuition fuzzy set.

Definition 2. Interval type-2 intuition fuzzy set $\widetilde{A}^{*}$ is expressed by membership function and nonmembership function defined as $\left[\underline{\mu}_{A}^{* *}(x, u), \bar{\mu}_{A}^{* *}(x, u)\right]$ and $\left[\underline{v}_{A}^{* *}(x, u)\right.$, $\left.\bar{v}_{\tilde{A}^{*}}(x, u)\right]$ for $x \in X$, and there are two FOUs defined as the following regarding the uncertainty of the membership function and nonmembership function. Then, the interval type- 2 intuition fuzzy set can be expressed as

$$
\begin{aligned}
\widetilde{A}^{*} & =\int_{x \in X} \int_{u \in J_{x}^{u}} \int_{v \in J_{x}^{v}} \frac{1}{(x, u)}=\int_{x \in X} \frac{\left[\int_{u \in J_{x}^{u}} \int_{v \in J_{x}^{v}}(1 / u)\right]}{x}, \\
\pi_{c}(x) & =\max \left(0,1-\left(\mu \widetilde{A}_{A}^{*}(x)+\underline{\nu}_{A}^{*}(x)\right)\right), \\
\bar{\pi}_{\mathrm{var}}(x) & =\max \left(0,1-\left(\bar{\mu}_{\bar{A}}^{*}(x)+\underline{\nu}_{A}^{*}(x)\right)\right), \\
\underline{\pi}_{\mathrm{var}}(x) & =\max \left(0,1-\left(\underline{\mu}_{A}^{*}(x)+\bar{\nu} \widetilde{\sim}^{*}(x)\right)\right), \quad 0 \leq \pi_{c}(x) \leq 1,0 \underline{\pi}_{\mathrm{var}}(x) \leq 1 .
\end{aligned}
$$

2.2. Brain Emotional Learning Network. One brain emotional learning network is composed of three parts including input, memory, and output. The memory part is inspired by the amygdala and orbitofrontal cortex functions, the former represents a sensory network and the latter is an emotional network in the memory space. The output of amygdala-like memory $a$ and orbitofrontal memory $o$ is defined as

$$
\begin{aligned}
& a=\chi \cdot \mathrm{SI}, \\
& o=\omega \cdot \mathrm{SI},
\end{aligned}
$$

where SI denotes the input of the network and $v$ and $w$ denote the gain of amygdala-like memory and orbitofrontal memory, respectively; then, the overall output of the brain emotional learning network is defined as

$$
u=a-o=(\chi-w) \cdot \mathrm{SI} .
$$

And, the learning rules of the sensory network and the emotional network are defined as

$$
d=b \cdot \mathrm{SI}+c \cdot u
$$




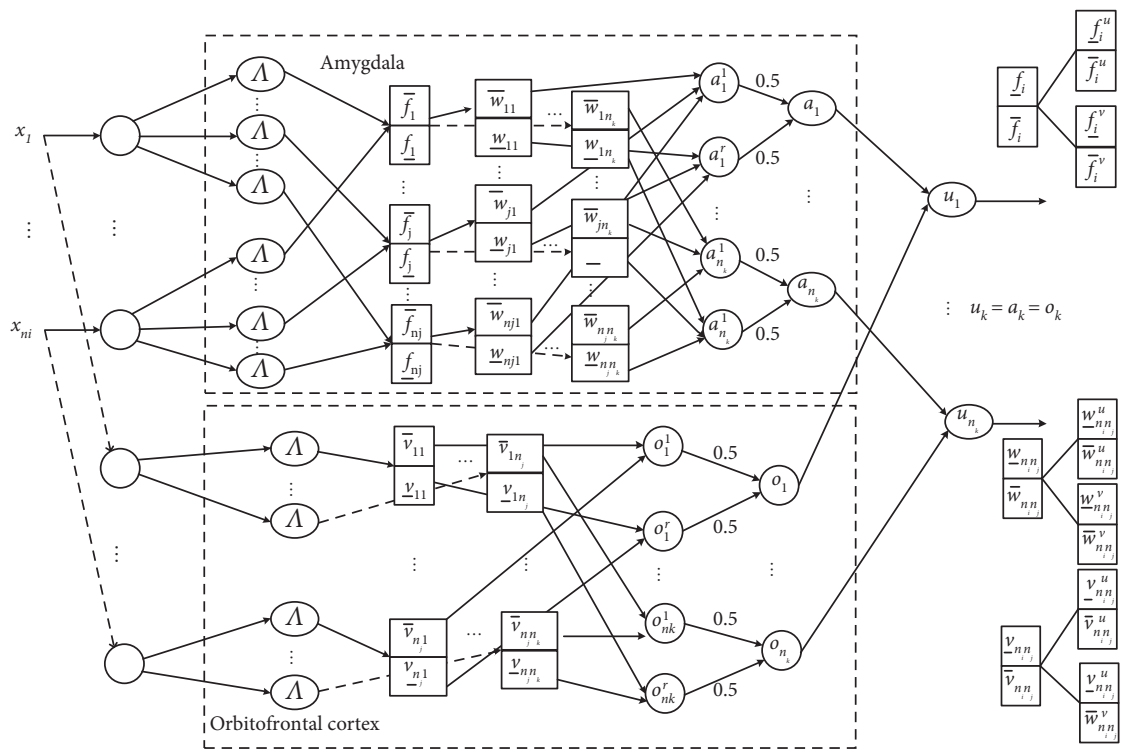

Figure 1: Architecture of the IT2IFBELN.

The parameters of $\alpha$ and $d$ represent the learning rate of the sensory network and emotional cue.

\section{The Interval Type-2 Intuition Fuzzy Brain Learning Network (IT2IFBELN)}

3.1. IT2IFBELN Structure. Figure 1 shows the structure of IT2IFBELN, which includes the amygdala network, the orbitofrontal cortex network, and the interval type- 2 intuition fuzzy sets. The proposed interval type- 2 intuition fuzzy brain emotional learning network (IT2IFBELN) is based on Professor Lin's articles about the interval type- 2 fuzzy brain emotional learning network. The difference between these two networks is that the intuition factors added in the current article at the third layer show as the spatial firing layer, and the following layers also have been changed. Then, the proposed IT2IFBELN is constructed with six layers, which include an input layer, an MF layer, a spatial firing layer, a weight memory layer, a summarily layer, and an output layer.

(1) The input space: the nodes in this space are given as $X=\left[x_{1}, x_{2}, \ldots, x_{n_{i}}\right]^{T}$, where $n_{i}$ represents the number of the input signals. All data from this layer will be transmitted to the next space without any computation.

(2) The MF space: in this layer, Gaussian activation function is adopted to finish the fuzzification by using interval type- 2 intuition membership function, which combined the membership and nonmembership functions together with the hesitancy index. As to the $i$ th input feature and the $k$ th rule, the upper and lower MFs with hesitation indices are given as

$$
\begin{aligned}
& \bar{\mu}_{i k}= \begin{cases}\exp \left(-\frac{\left(x_{i}-c_{i k}^{l}\right)^{2}}{2 \sigma_{i k}^{2}}\right)\left(1-\bar{\pi}_{c, i k}\left(x_{i}\right)\right), & x_{i}(t) \leq c_{i k}^{l}, \\
1, & c_{i k}^{l} \leq x_{i}(t) \leq c_{i k}^{r}, \\
\exp \left(-\frac{\left(x_{i}-c_{i k}^{r}\right)^{2}}{2 \sigma_{i k}^{2}}\right)\left(1-\underline{\pi}_{c, i k}\left(x_{i}\right)\right), & x_{i}(t) \geq c_{i k}^{r},\end{cases} \\
& \underline{\mu}_{i k}= \begin{cases}\exp \left(-\frac{\left(x_{i}-c_{i k}^{r}\right)^{2}}{2 \sigma_{i k}^{2}}\right)\left(1-\bar{\pi}_{c, i k}\left(x_{i}\right)\right), & x_{i}(t) \leq \frac{c_{i k}^{l}+c_{i k}^{r}}{2}, \\
\exp \left(-\frac{\left(x_{i}-c_{i k}^{l}\right)^{2}}{2 \sigma_{i k}^{2}}\right)\left(1-\bar{\pi}_{c, i k}\left(x_{i}\right)\right), & x_{i}(t) \geq \frac{c_{i k}^{l}+c_{i k}^{r}}{2} .\end{cases}
\end{aligned}
$$


Similarly, upper and lower non-MFs with hesitation indices could be given as

$$
\begin{aligned}
& \bar{v}_{i k}= \begin{cases}1-\pi_{\mathrm{var}, i k}\left(x_{i}\right)-\exp \left(-\frac{\left(x_{i}-c_{i k}^{r}\right)^{2}}{2 \sigma_{i k}^{2}}\right)\left(1-\bar{\pi}_{c, i k}\left(x_{i}\right)\right), & x_{i}(t) \leq \frac{c_{i k}^{l}+c_{i k}^{r}}{2}, \\
1-\pi_{\mathrm{var}, i k}\left(x_{i}\right)-\exp \left(-\frac{\left(x_{i}-c_{i k}^{l}\right)^{2}}{2 \sigma_{i k}^{2}}\right)\left(1-\bar{\pi}_{c, i k}\left(x_{i}\right)\right), & x_{i}(t)>\frac{c_{i k}^{l}+c_{i k}^{r}}{2},\end{cases} \\
& \underline{v}_{i k}= \begin{cases}1-\pi_{\mathrm{var}, i k}\left(x_{i}\right)-\exp \left(-\frac{\left(x_{i}-c_{i k}^{l}\right)^{2}}{2 \sigma_{i k}^{2}}\right)\left(1-\underline{\pi}_{c, i k}\left(x_{i}\right)\right), & x_{i}(t)<c_{i k}^{l}, \\
0, & c_{i k}^{l}<x_{i}(t)<c_{i k}^{r}, \\
1-\pi_{\mathrm{var}, i k}\left(x_{i}\right)-\exp \left(-\frac{\left(x_{i}-c_{i k}^{r}\right)^{2}}{2 \sigma_{i k}^{2}}\right)\left(1-\underline{\pi}_{c, i k}\left(x_{i}\right)\right), & x_{i}(t)>c_{i k}^{r},\end{cases}
\end{aligned}
$$

where $\pi_{\mathrm{var}, i k}$ and $\pi_{c, i k}$ indicate the hesitation level of variance and center, respectively. The parameters $c_{i k}^{l}, c_{i k}^{r}$, and $\sigma_{i k}$ represent the center and variance of the fuzzy rules for the $i$ th input feature and the $k$ th rule. Then, the output of the ith input feature and the $k$ th rule can be represented by an interval MF $\left[\underline{\mu}_{i k}, \bar{\mu}_{i k}\right]$ and an interval non-MF $\left[\underline{v}_{i k}, \bar{v}_{i k}\right]$.
(3) Spatial firing layer: the rule in this layer is composed of both upper and lower firing strength of MFs as well as non-MFs. The firing strength of the interval type-2 intuition fuzzy rule is the interval and can be calculated as the following:

$$
\left\{\begin{array}{l}
F_{k}^{\mu}=\left[\underline{f}_{k}^{\mu}, \underline{f}_{k}^{\mu}\right], \underline{f}_{k}^{\mu}=\prod_{i=1}^{m} \underline{\mu}_{i k}, \bar{f}_{k}^{\mu}=\prod_{i=1}^{m} \bar{\mu}_{i k}, \quad k=1,2, \ldots, M, \\
F_{k}^{v}=\left[\underline{f}_{k}^{v}, \bar{f}_{k}^{v}\right], \underline{f}_{k}^{v}=\prod_{i=1}^{m} \underline{v}_{i k}, \bar{f}_{k}^{v}=\prod_{i=1}^{m} \bar{v}_{i k}, \quad k=1,2, \ldots, M,
\end{array}\right.
$$

where $F_{k}^{v}$ and $F_{k}^{\mu}$ denote the intervals of the firing strength of MFs and non-MFs and $m$ and $M$ represent the number of input signals and fuzzy rules, respectively.

(4) Weight memory space: this layer contains two memory spaces, and they are amygdala memory and orbitofrontal memory, which are interval values because the firing space is the interval. Thus, the weight of the $i$ th rule and the $k$ th output of the amygdala network and orbitofrontal network is given as

$$
\begin{aligned}
\omega_{k}^{\mu} & =\left[\underline{\omega}_{k}^{\mu}, \bar{\omega}_{k}^{\mu}\right], \\
\omega_{k}^{v} & =\left[\underline{\omega}_{k}^{v}, \bar{\omega}_{k}^{v}\right], \\
\chi_{k}^{\mu} & =\left[\underline{\chi}_{k}^{\mu}, \bar{\chi}_{k}^{\mu}\right], \\
\chi_{k}^{v} & =\left[\underline{\chi}_{k}^{v}, \bar{\chi}_{k}^{v}\right] .
\end{aligned}
$$


(5) Defuzzification space: the output of this defuzzification space is calculated by the output of the firing space and the weight space. So, the left- and right- most point values for amygdala and orbitofrontal network outputs are as the following:

$$
\begin{aligned}
& a_{k}^{l}=\frac{\beta_{\mathrm{jal}} \underline{f}_{k}^{\mu} \underline{w_{k}^{\mu}}+\left(1-\beta_{\mathrm{jal}}\right) \underline{f}_{k}^{v} \underline{w_{k}^{v}}}{\beta_{\mathrm{jal}} \sum_{j=1}^{n_{j}} \underline{f}_{k}^{\mu}+\left(1-\beta_{\mathrm{jal}}\right) \sum_{j=1}^{n_{j}} \underline{f}_{k}^{v}}=\frac{\beta_{\mathrm{jal}} \prod_{i=1}^{M} \underline{\mu}_{i k} \underline{w}_{k}^{\mu}+\left(1-\beta_{\mathrm{jal}}\right) \prod_{i=1}^{M} \underline{v}_{i k} \underline{w}_{k}^{v}}{\beta_{\mathrm{jal}} \prod_{i=1}^{M} \underline{\mu}_{i k}+\left(1-\beta_{\mathrm{jal}}\right) \prod_{i=1}^{M} \underline{v}_{i k}}, \\
& a_{k}^{r}=\frac{\beta_{\mathrm{jar}} \bar{f}_{k}^{\mu} \bar{\omega}_{k}^{\mu}+\left(1-\beta_{\mathrm{jar}}\right) \bar{f}_{k}^{v} \bar{\omega}_{k}^{v}}{\beta_{\mathrm{jar}} \sum_{j=1}^{n_{j}} \bar{f}_{k}^{\mu}+\left(1-\beta_{\mathrm{jar}}\right) \sum_{j=1}^{n_{j}} \bar{f}_{k}^{v}}=\frac{\beta_{\mathrm{jar}} \prod_{i=1}^{M} \bar{\mu}_{i k} \bar{\omega}_{k}^{\mu}+\left(1-\beta_{\mathrm{jar}}\right) \prod_{i=1}^{M} \bar{v}_{i k} \bar{\omega}_{k}^{v}}{\beta_{\mathrm{jar}} \prod_{i=1}^{M} \bar{\mu}_{i k}+\left(1-\beta_{\mathrm{jar}}\right) \prod_{i=1}^{M} \bar{v}_{i k}}, \\
& o_{k}^{l}=\frac{\beta_{\mathrm{jol}} \underline{f}_{k}^{\mu} \underline{\chi}_{k}^{\mu}+\left(1-\beta_{\mathrm{jol}}\right) \underline{f}_{k}^{v} \underline{\chi}_{k}^{v}}{\beta_{\mathrm{jol}} \sum_{j=1}^{n_{j}} \underline{f}_{k}^{\mu}+\left(1-\beta_{\mathrm{jol}}\right) \sum_{j=1}^{n_{j}} \underline{f}_{k}^{v}}=\frac{\beta_{\mathrm{jol}} \prod_{i=1}^{M} \underline{\mu}_{i k} \underline{\phi}_{k}^{\mu}+\left(1-\beta_{\mathrm{jol}}\right) \prod_{i=1}^{M} \underline{v}_{i k} \underline{\chi}_{k}^{v}}{\beta_{\mathrm{jol}} \prod_{i=1}^{M} \underline{\mu}_{i k}+\left(1-\beta_{\mathrm{jol}}\right) \prod_{i=1}^{M} \underline{v}_{i k}}, \\
& o_{k}^{r}=\frac{\beta_{\mathrm{jor}} \bar{f}_{k}^{\mu} \bar{\chi}_{k}^{\mu}+\left(1-\beta_{\mathrm{jor}}\right) \bar{f}_{k}^{v} \bar{\chi}_{k}^{v}}{\beta_{\mathrm{jor}} \sum_{j=1}^{n_{j}} \bar{f}_{k}^{\mu}+\left(1-\beta_{\mathrm{jor}}\right) \sum_{j=1}^{n_{j}} \bar{f}_{k}^{v}}=\frac{\beta_{\mathrm{jor}} \prod_{i=1}^{M} \bar{\mu}_{i k} \bar{\chi}_{k}^{\mu}+\left(1-\beta_{\mathrm{jor}}\right) \prod_{i=1}^{M} \bar{v}_{i k} \bar{\chi}_{k}^{v}}{\beta_{\mathrm{jor}} \prod_{i=1}^{M} \bar{\mu}_{i k}+\left(1-\beta_{\mathrm{jor}}\right) \prod_{i=1}^{M} \bar{v}_{i k}},
\end{aligned}
$$

where parameters $\beta_{\mathrm{jar}}, \beta_{\mathrm{jal}}, \beta_{\mathrm{jor}}, \beta_{\mathrm{jol}} \in[0,1]$ indicate the contribution of MFs and non-MFs values for the output values, when $\beta_{\mathrm{jar}}=0, \beta_{\mathrm{jal}}=0, \beta_{\mathrm{jol}}=0$, and $\beta_{\text {jor }}=0$. The non-MFs have impact on the output results, and then $\beta_{\text {jar }}=1, \beta_{\text {jal }}=1, \beta_{\text {jol }}=1$, and $\beta_{\text {jor }}=$ 1 means that the non-MFs have no influence on the output results.

(6) Output space: the output of the defuzzification space is an interval value, so the average operations are used to obtain the amygdala network and $e$ orbitofrontal cortex network outputs as

$$
\begin{gathered}
a_{k}=\frac{a_{k}^{r}+a_{k}^{l}}{2}, \\
o_{k}=\frac{o_{k}^{r}+o_{k}^{l}}{2} .
\end{gathered}
$$

3.2. Self-Organizing Algorithm for IT2IFBELN. To achieve the better structure of the IT2IFBELN, it requires to decide the appropriate rules by using the corresponding self-organizing algorithm. If the number of rules is large, it will cost long time for computational loading, and if the numbers are small, it cannot reflect all the cases especially when the data have large ranges. Initially, there are no rules and MFs in the first space, and when the first input data stream comes, the first MF will be created. Then, the self-organizing algorithm will be used to determine whether to generate new rules and MFs or to delete inappropriate rules and MFs. In this paper, the (interval type-2 fuzzy c-means) IT2FCM is used to choose the cluster centers of the membership functions for fuzzy rules of the RT2WFNN. The IT2FCM [29] is an iterative optimization algorithm that minimizes the objective function as

$$
\begin{aligned}
J_{m}(U, V)= & \sum_{i=1}^{c} \sum_{k=1}^{n} \mu_{i k}(m) d_{i k}^{2} \\
& \cdot\left\{\begin{array}{l}
\bar{\mu}_{i}(k)=\max \left(\frac{1}{\sum_{j=1}^{c}\left(d_{k} / d_{j}\right)^{\left(2 /\left(m_{1}-1\right)\right)}}, \frac{1}{\sum_{j=1}^{c}\left(d_{i k} / d_{j k}\right)^{\left(2 /\left(m_{2}-1\right)\right)}}\right) \\
\underline{\mu}_{i}(k)=\min \left(\frac{1}{\sum_{j=1}^{c}\left(d_{i k} / d_{j k}\right)^{\left(2 /\left(m_{1}-1\right)\right)}}, \frac{1}{\sum_{j=1}^{c}\left(d_{i k} / d_{j k}\right)^{\left(2 /\left(m_{2}-1\right)\right)}}\right)
\end{array}\right\},
\end{aligned}
$$


where $d_{i k}^{2}=\left\|x_{k}-v_{i}\right\|$ denotes the distance between the cluster centers $v_{i}$ and input pattern $x_{k}$. The main steps of the IT2FCM can be shown as follows:

(1) Setting the fuzzifiers and the number $c$ of the cluster prototypes and initializing the cluster center $V$ using the GA algorithm

(2) Calculating the distance between the cluster centers $v_{i}$ and input pattern $x_{k}$, the lower and upper partition functions can be calculated by equation (2)

(3) By updating the cluster center $V^{\prime}$, the interval type- 1 fuzzy set $\left[c_{L}, c_{R}\right]$ is obtained during the iterative process and the optimal improved EKM algorithm, which is adopted to estimate both ends of the interval fuzzy set

(4) The new cluster center $V^{\prime}$ is updated by a defuzzification method as $V^{\prime}=\left(c_{L}+c_{R}\right) / 2$, and go to the next step, otherwise set $V=V^{\prime}$

(5) Finally, the type reduction of the type-2 fuzzy partition matrix is set as $\mu_{i k}=\left(\mu_{i k}^{L}+\mu_{i k}^{R}\right) / 2$

The output of the IT2FCM algorithm is an interval type-2 FS that cannot be transformed to a crisp set by the defuzzifier directly. Hence, the type-reduction process is needed. The aim of type reduction is to compute the centroid of a type-2 fuzzy set. At present, the iterative Karnik-Mendel (KM) algorithm and the enhanced Karnik-Mendel (EKM) algorithm can compute the centroid of an interval type- 2 fuzzy set efficiently, and here, the improved EKM is used, which could change the initialization conditions of switch points and improve the searching method for switch points.

3.3. Parameter Learning Algorithm for IT2IFBELN. The online tuning laws for the parameters of IT2FIBELN are given as

$$
\begin{aligned}
& \underline{\hat{\omega}}_{j k}^{u}(t+1)=\underline{\widehat{\omega}}_{j k}^{u}(t)-\widehat{\eta}_{\omega} \frac{\partial s(t) \dot{s}(t)}{\partial \underline{\widehat{\omega}}_{j k}^{u}}, \\
& \overline{\bar{\omega}}_{j k}^{u}(t+1)=\overline{\bar{\omega}}_{j k}^{u}(t)-\widehat{\eta}_{\omega} \frac{\partial s(t) \dot{s}(t)}{\partial \overline{\bar{\omega}}_{j k}^{u}}, \\
& \underline{\widehat{\omega}}_{j k}^{v}(t+1)=\underline{\widehat{\omega}}_{j k}^{v}(t)-\hat{\eta}_{\omega} \frac{\partial s(t) \dot{s}(t)}{\partial \underline{\widehat{\omega}}_{j k}^{v}}, \\
& \overline{\bar{\omega}}_{j k}^{v}(t+1)=\overline{\bar{\omega}}_{j k}^{v}(t)-\bar{\eta}_{\omega} \frac{\partial s(t) \dot{s}(t)}{\partial \overline{\bar{\omega}}_{j k}^{v}}, \\
& \overline{\bar{c}}_{i j}^{a}(t+1)=\overline{\bar{c}}_{i j}^{a}(t)-\hat{\eta}_{c} \frac{\partial s(t) \dot{s}(t)}{\partial \overline{\bar{c}}_{i j}^{a}},
\end{aligned}
$$

$$
\begin{aligned}
& \widehat{\underline{c}}_{i j}^{a}(t+1)=\underline{\hat{c}}_{i j}^{a}(t)-\hat{\eta}_{c}^{u} \frac{\partial s(t) \dot{s}(t)}{\partial \underline{\underline{c}}_{i j}^{a}}, \\
& \hat{\sigma}_{i j}^{a}(t+1)=\hat{\sigma}_{i j}^{a}(t)-\hat{\eta}_{\sigma} \frac{s(t) \dot{s}(t)}{\partial \hat{\sigma}_{i j}^{a}}, \\
& \hat{\pi}_{\mathrm{var}, i j k}^{a}(t+1)=\hat{\pi}_{\mathrm{var}, i j k}^{a}(t)-\hat{\eta}_{\mathrm{var}, \pi} \frac{\partial s(t) \dot{s}(t)}{\partial \hat{\pi}_{\mathrm{var}, i j k}^{a}}, \\
& \overline{\bar{\pi}}_{c, i j k}^{a}(t+1)=\overline{\bar{\pi}}_{c, i j k}^{a}(t)-\widehat{\eta}_{c, \pi} \frac{\partial s(t) \dot{s}(t)}{\partial \overline{\bar{\pi}}_{c, i j k}^{a}}, \\
& \underline{\tilde{\pi}}_{c, i j k}^{a}(t+1)=\underline{\tilde{\pi}}_{c, i j k}^{a}(t)-\widehat{\eta}_{c, \pi} \frac{\partial s(t) \dot{s}(t)}{\partial \underline{\pi}_{c, i j k}^{a}}, \\
& \underline{\hat{\chi}}_{j k}^{u}(t+1)=\underline{\bar{\chi}}_{j k}^{u}(t)-\bar{\eta}_{\chi} \frac{\partial s(t) \dot{s}(t)}{\partial \underline{\bar{\chi}}_{j k}^{u}}, \\
& \overline{\bar{\chi}}_{j k}^{u}(t+1)=\overline{\bar{\chi}}_{j k}^{u}(t)-\widehat{\eta}_{\chi} \frac{\partial s(t) \dot{s}(t)}{\partial \overline{\bar{\chi}}_{j k}^{u}}, \\
& \underline{\chi}_{j k}^{v}(t+1)=\underline{\chi}_{j k}^{v}(t)-\bar{\eta}_{\chi} \frac{\partial s(t) \dot{s}(t)}{\partial \underline{\chi}_{j k}^{v}}, \\
& \overline{\bar{\chi}}_{j k}^{v}(t+1)=\widehat{\bar{\chi}}_{j k}^{v}(t)-\widehat{\eta}_{\chi} \frac{\partial s(t) \dot{s}(t)}{\partial \overline{\bar{\chi}}_{j k}^{v}}, \\
& \widehat{\bar{c}}_{i j}^{o}(t+1)=\overline{\bar{c}}_{i j}^{o}(t)-\widehat{\eta}_{c} \frac{\partial s(t) \dot{s}(t)}{\partial \overline{\bar{c}}_{i j}^{o}}, \\
& \widehat{\underline{c}}_{i j}^{o}(t+1)=\underline{\underline{c}}_{i j}^{o}(t)-\widehat{\eta}_{c} \frac{\partial s(t) \dot{s}(t)}{\partial \underline{\hat{c}}_{i j}^{o}}, \\
& \widehat{\sigma}_{i j}^{o}(t+1)=\widehat{\sigma}_{i j}^{o}(t)-\widehat{\eta}_{\sigma} \frac{s(t) \dot{s}(t)}{\partial \hat{\sigma}_{i j}^{o}},
\end{aligned}
$$

where $\eta_{\omega}$ and $\mu_{\chi}$ represent the learning rates for updating the weight of the orbitofrontal cortex and an amygdala network, respectively, $\eta_{c}$ and $\mu_{\sigma}$ represent the learning rates for updating the means and the variances of type-2 Gaussian MFs, respectively, and $\eta_{c, \pi}$ and $\mu_{\mathrm{var}, \pi}$ represent the learning rates for updating the IF indices which indicate the hesitation level of the variance and center. By applying the chain rule for the derivation of the above terms, we get 


$$
\begin{aligned}
& \frac{\partial s(t) \dot{s}(t)}{\partial \underline{\hat{\omega}}_{j k}^{u}}=\frac{\partial s(t) \dot{s}(t)}{\partial \widehat{u}_{\mathrm{IT} \mathrm{FiBELN}_{k}}} \frac{\partial \widehat{u}_{\mathrm{IT} 2 \mathrm{FiBELN}_{k}}}{\partial a_{k}} \frac{\partial a_{k}}{\partial a_{k}^{l}} \frac{\partial a_{k}^{l}}{\partial \underline{\hat{\omega}}_{j k}^{u}}=-\frac{1}{2} s(t) \frac{\beta_{\mathrm{jal}} \underline{f}_{k}^{\mu}}{\beta_{\mathrm{jal}} \sum_{j=1}^{n_{j}} \underline{f}_{k}^{\mu}+\left(1-\beta_{\mathrm{jal}}\right) \sum_{j=1}^{n_{j}} \underline{f}_{k}^{v}}, \\
& \frac{\partial s(t) \dot{s}(t)}{\partial \widehat{\bar{\omega}}_{j k}^{u}}=\frac{\partial s(t) \dot{s}(t)}{\partial \widehat{u}_{\text {IT2FIBELN }_{k}}} \frac{\partial \widehat{u}_{\widehat{u}_{\text {T2FIBELIN }}}}{\partial a_{k}} \frac{\partial a_{k}}{\partial a_{k}^{r}} \frac{\partial a_{k}^{r}}{\partial \overline{\bar{\omega}}_{j k}^{u}}=-\frac{1}{2} s(t) \frac{\beta_{\text {jar }} \bar{f}_{k}^{u}}{\beta_{\text {jar }} \sum_{j=1}^{n_{j}} \bar{f}_{k}^{\mu}+\left(1-\beta_{\text {jar }}\right) \sum_{j=1}^{n_{j}} \bar{f}_{k}^{v}}, \\
& \frac{\partial s(t) \dot{s}(t)}{\partial \underline{\omega}_{j k}^{v}}=\frac{\partial s(t) \dot{s}(t)}{\partial \widehat{u}_{\mathrm{TT}_{2 \mathrm{FiBELN}}}} \frac{\partial \widehat{u}_{\mathrm{TT}_{k} \mathrm{FiBELN}_{k}}}{\partial a_{k}} \frac{\partial a_{k}}{\partial a_{k}^{l}} \frac{\partial a_{k}^{l}}{\partial \underline{\omega}_{j k}^{v}}=-\frac{1}{2} s(t) \frac{\left(1-\beta_{\mathrm{jal}}\right) \underline{f}_{k}^{v}}{\beta_{\mathrm{jal}} \sum_{j=1}^{n_{j}} \underline{f}_{k}^{\mu}+\left(1-\beta_{\mathrm{jal}}\right) \sum_{j=1}^{n_{j}} \underline{f}_{k}^{v}}, \\
& \frac{\partial s(t) \dot{s}(t)}{\partial \widehat{\bar{\omega}}_{j k}^{v}}=\frac{\partial s(t) \dot{s}(t)}{\partial \widehat{u}_{\text {IT2FIBELN }_{k}}} \frac{\partial \widehat{u}_{\text {TT2FIBELN }_{k}}}{\partial a_{k}} \frac{\partial a_{k}}{\partial a_{k}^{r}} \frac{\partial a_{k}^{r}}{\partial \overline{\bar{\omega}}_{j k}^{v}}=-\frac{1}{2} s(t) \frac{\left(1-\beta_{\text {jar }}\right) \bar{f}_{k}^{v}}{\beta_{\text {jar }} \sum_{j=1}^{n_{j}} \bar{f}_{k}^{\mu}+\left(1-\beta_{\mathrm{jar}}\right) \sum_{j=1}^{n_{j}} \bar{f}_{k}^{v}}, \\
& \frac{\partial s(t) \dot{s}(t)}{\partial \widehat{\sigma}_{i j}^{a}}=\sum_{k=1}^{n_{k}}\left(\frac{\partial s(t) \dot{\mathcal{s}}(t)}{\partial \widehat{u}_{\text {IT2FIBELN }_{k}}} \frac{\partial \widehat{u}_{\text {IT2FIBELN }_{k}}}{\partial a_{k}^{l}}\left(\frac{\partial a_{k}}{\partial a_{k}^{l}} \frac{\partial a_{k}^{l}}{\partial f_{j}^{l}} \frac{\partial f_{j}^{l}}{\partial \widehat{\sigma}_{i j}^{a}}+\frac{\partial a_{k}}{\partial a_{k}^{r}} \frac{\partial a_{k}^{r}}{\partial f_{j}^{r}} \frac{\partial f_{j}^{r}}{\partial \widehat{\sigma}_{i j}^{a}}\right)\right)
\end{aligned}
$$

$$
=-\frac{1}{2} s(t) \sum_{k=1}^{n_{k}}\left(\begin{array}{c}
\frac{\beta_{\mathrm{jal}} \underline{\omega}_{j k}^{u}-a_{k}^{l}}{\beta_{\mathrm{jal}} \sum_{j=1}^{n_{j}} \underline{f}_{k}^{\mu}+\left(1-\beta_{\mathrm{jal}}\right) \sum_{j=1}^{n_{j}} \underline{f}_{k}^{v}} \frac{\partial \underline{f}_{j}^{u}}{\partial \bar{\sigma}_{i j}^{a}}+\frac{\left(1-\beta_{\mathrm{jal}}\right) \underline{\omega}_{j k}^{v}-a_{k}^{l}}{\beta_{\mathrm{jal}} \sum_{j=1}^{n_{j}} \underline{f}_{k}^{\mu}+\left(1-\beta_{\mathrm{jal}}\right) \sum_{j=1}^{n_{j}} \underline{f}_{k}^{v}} \frac{\partial \underline{f}_{j}^{v}}{\partial \bar{\sigma}_{i j}^{a}} \\
+\frac{\beta_{\mathrm{jar}} \bar{\omega}_{j k}^{u}-a_{k}^{r}}{\beta_{\mathrm{jar}} \sum_{j=1}^{n_{j}} \bar{f}_{k}^{\mu}+\left(1-\beta_{\mathrm{jar}}\right) \sum_{j=1}^{n_{j}} \bar{f}_{k}^{v}} \frac{\partial \bar{f}_{j}^{u}}{\partial \bar{\sigma}_{i j}^{a}}+\frac{\left(1-\beta_{\mathrm{jar}}\right) \bar{\omega}_{j k}^{v}-a_{k}^{r}}{\beta_{\mathrm{jar}} \sum_{j=1}^{n_{j}} \bar{f}_{k}^{\mu}+\left(1-\beta_{\mathrm{jar}}\right) \sum_{j=1}^{n_{j}} \bar{f}_{k}^{v}} \frac{\partial \bar{f}_{j}^{v}}{\partial \hat{\sigma}_{i j}^{a}}
\end{array}\right),
$$

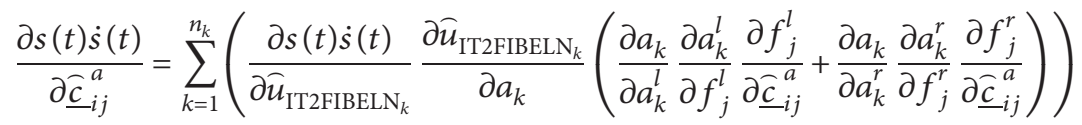

$$
=-\frac{1}{2} s(t) \sum_{k=1}^{n_{k}}\left(\begin{array}{c}
\frac{\beta_{\mathrm{jal}} \underline{\omega}_{j k}^{u}-a_{k}^{l}}{\beta_{\mathrm{jal}} \sum_{j=1}^{n_{j}} \underline{f}_{k}^{\mu}+\left(1-\beta_{\mathrm{jal}}\right) \sum_{j=1}^{n_{j}} \underline{f}_{k}^{v}} \frac{\partial \underline{f}_{j}^{u}}{\partial \underline{\bar{c}}_{i j}^{a}}+\frac{\left(1-\beta_{\mathrm{jal}}\right) \underline{\omega}_{j k}^{v}-a_{k}^{l}}{\beta_{\mathrm{jal}} \sum_{j=1}^{n_{j}} \underline{f}_{k}^{\mu}+\left(1-\beta_{\mathrm{jal}}\right) \sum_{j=1}^{n_{j}} \underline{f}_{k}^{v}} \frac{\partial \underline{f}_{j}^{v}}{\partial \underline{\bar{c}}_{i j}^{a}} \\
+\frac{\beta_{\mathrm{jar}} \bar{\omega}_{j k}^{u}-a_{k}^{r}}{\beta_{\mathrm{jar}} \sum_{j=1}^{n_{j}} \bar{f}_{k}^{\mu}+\left(1-\beta_{\mathrm{jar}}\right) \sum_{j=1}^{n_{j}} \bar{f}_{k}^{v}} \frac{\partial \bar{f}_{j}^{u}}{\partial \underline{\hat{c}}_{i j}^{a}}+\frac{\left(1-\beta_{\mathrm{jar}}\right) \bar{\omega}_{j k}^{v}-a_{k}^{r}}{\beta_{\mathrm{jar}} \sum_{j=1}^{n_{j}} \bar{f}_{k}^{\mu}+\left(1-\beta_{\mathrm{jar}}\right) \sum_{j=1}^{n_{j}} \bar{f}_{k}^{v}} \frac{\partial \bar{f}_{j}^{v}}{\partial \underline{\hat{c}}_{i j}^{a}}
\end{array}\right),
$$

$$
\frac{\partial s(t) \dot{s}(t)}{\partial \underline{\chi}_{j k}^{u}}=\frac{\partial s(t) \dot{s}(t)}{\partial \widehat{u}_{\mathrm{IT} 2 \mathrm{FiBELN}_{k}}} \frac{\partial \bar{u}_{\mathrm{TT2FiBELN}_{k}}}{\partial o_{k}} \frac{\partial o_{k}}{\partial o_{k}^{l}} \frac{\partial o_{k}^{l}}{\partial \underline{\chi}_{j k}^{u}}=-\frac{1}{2} s(t) \frac{\beta_{\mathrm{jol}} \underline{f}_{k}^{\mu}}{\beta_{\mathrm{jol}} \sum_{j=1}^{n_{j}} \underline{f}_{k}^{\mu}+\left(1-\beta_{\mathrm{jol}}\right) \sum_{j=1}^{n_{j}} \underline{f}_{k}^{v}},
$$$$
\frac{\partial s(t) \dot{s}(t)}{\partial \widehat{\bar{\chi}}_{j k}^{u}}=\frac{\partial s(t) \dot{s}(t)}{\partial \widehat{u}_{\text {TT2FIBELN }_{k}}} \frac{\partial \widehat{u}_{\bar{u}_{\text {TRFIIELI }}}}{\partial o_{k}} \frac{\partial o_{k}}{\partial o_{k}^{r}} \frac{\partial o_{k}^{r}}{\partial \widehat{\bar{\chi}}_{j k}^{u}}=-\frac{1}{2} s(t) \frac{\beta_{\text {jor }} \bar{f}_{k}^{u}}{\beta_{\text {jor }} \sum_{j=1}^{n_{j}} \bar{f}_{k}^{\mu}+\left(1-\beta_{\text {jor }}\right) \sum_{j=1}^{n_{j}} \bar{f}_{k}^{v}},
$$$$
\frac{\partial s(t) \dot{s}(t)}{\partial \hat{\chi}_{j k}^{v}}=\frac{\partial s(t) \dot{s}(t)}{\partial \hat{u}_{\text {IT2FiBELN }_{k}}} \frac{\partial \widehat{u}_{\text {TT2FiBELN }_{k}}}{\partial o_{k}} \frac{\partial o_{k}}{\partial o_{k}^{l}} \frac{\partial o_{k}^{l}}{\partial \underline{\chi}_{j k}^{v}}=-\frac{1}{2} s(t) \frac{\left(1-\beta_{\text {jol }}\right) \underline{f}_{k}^{v}}{\beta_{\text {jol }} \sum_{j=1}^{n_{j}} \underline{f}_{k}^{\mu}+\left(1-\beta_{\text {jol }}\right) \sum_{j=1}^{n_{j}} \underline{f}_{k}^{v}},
$$$$
\frac{\partial s(t) \dot{s}(t)}{\partial \overline{\bar{\chi}}_{j k}^{v}}=\frac{\partial s(t) \dot{s}(t)}{\partial \bar{u}_{\text {TT2FIBELN }_{k}}} \frac{\partial \bar{u}_{\overline{\mathrm{ITTFFBELIN}}_{k}}}{\partial o_{k}} \frac{\partial a_{k}}{\partial o_{k}^{r}} \frac{\partial o_{k}^{r}}{\partial \overline{\bar{\chi}}_{j k}^{v}}=-\frac{1}{2} s(t) \frac{\left(1-\beta_{\text {jor }}\right) \bar{f}_{k}^{v}}{\beta_{\text {jor }} \sum_{j=1}^{n_{j}} \bar{f}_{k}^{\mu}+\left(1-\beta_{\text {jor }}\right) \sum_{j=1}^{n_{j}} \bar{f}_{k}^{v}},
$$ 


$$
\begin{aligned}
& \frac{\partial s(t) \dot{s}(t)}{\partial \widehat{\sigma}_{i j}^{o}}=\sum_{k=1}^{n_{k}}\left(\frac{\partial s(t) \dot{s}(t)}{\partial \widehat{u}_{\text {IT2FIBELN }_{k}}} \frac{\partial \widehat{u}_{\text {IT2FIBELN }_{k}}}{\partial o_{k}^{l}}\left(\frac{\partial o_{k}}{\partial o_{k}^{l}} \frac{\partial o_{k}^{l}}{\partial f_{j}^{l}} \frac{\partial f_{j}^{l}}{\partial \widehat{\sigma}_{i j}^{o}}+\frac{\partial o_{k}}{\partial o_{k}^{r}} \frac{\partial o_{k}^{r}}{\partial f_{j}^{r}} \frac{\partial f_{j}^{r}}{\partial \widehat{\sigma}_{i j}^{o}}\right)\right) \\
& =-\frac{1}{2} s(t) \sum_{k=1}^{n_{k}}\left(\begin{array}{c}
\frac{\beta_{\text {jol }} \underline{x}_{j k}^{u}-o_{k}^{l}}{\beta_{\text {jol }} \sum_{j=1}^{n_{j}} \underline{f}_{k}^{\mu}+\left(1-\beta_{\text {jol }}\right) \sum_{j=1}^{n_{j}} \underline{f}_{k}^{v}} \frac{\partial \underline{f}_{j}^{u}}{\partial \bar{\sigma}_{i j}^{o}}+\frac{\left(1-\beta_{\text {jol }}\right) \underline{\chi}_{j k}^{v}-o_{k}^{l}}{\beta_{\text {jol }} \sum_{j=1}^{n_{j}} \underline{f}_{k}^{\mu}+\left(1-\beta_{\text {jol }}\right) \sum_{j=1}^{n_{j}} \underline{f}_{k}^{v}} \frac{\partial \underline{f}_{j}^{v}}{\partial \vec{\sigma}_{i j}^{o}} \\
+\frac{\beta_{\text {jor }} \bar{\chi}_{j k}^{u}-o_{k}^{r}}{\beta_{\text {jor }} \sum_{j=1}^{n_{j}} \bar{f}_{k}^{\mu}+\left(1-\beta_{j o r}\right) \sum_{j=1}^{n_{j}} \bar{f}_{k}^{v}} \frac{\partial \bar{f}_{j}^{u}}{\partial \bar{\sigma}_{i j}^{o}}+\frac{\left(1-\beta_{\text {jor }}\right) \bar{\chi}_{j k}^{v}-o_{k}^{r}}{\beta_{\text {jor }} \sum_{j=1}^{n_{j}} \bar{f}_{k}^{\mu}+\left(1-\beta_{\text {jor }}\right) \sum_{j=1}^{n_{j}} \bar{f}_{k}^{v}} \frac{\partial \bar{f}_{j}^{v}}{\partial \bar{\sigma}_{i j}^{o}}
\end{array}\right), \\
& \frac{\partial s(t) \dot{s}(t)}{\partial \widehat{\bar{c}}_{i j}^{o}}=\sum_{k=1}^{n_{k}}\left(\frac{\partial s(t) \dot{s}(t)}{\partial \hat{u}_{\text {TT2FIBELN }_{k}}} \frac{\partial \widehat{u}_{\text {IT2FIBELN }_{k}}}{\partial o_{k}}\left(\frac{\partial o_{k}}{\partial o_{k}^{l}} \frac{\partial o_{k}^{l}}{\partial f_{j}^{l}} \frac{\partial f_{j}^{l}}{\partial \widehat{\bar{c}}_{i j}^{o}}+\frac{\partial a_{k}}{\partial o_{k}^{r}} \frac{\partial o_{k}^{r}}{\partial f_{j}^{r}} \frac{\partial f_{j}^{r}}{\partial \widehat{\bar{c}}_{i j}^{o}}\right)\right) \\
& =-\frac{1}{2} s(t) \sum_{k=1}^{n_{k}}\left(\begin{array}{c}
\frac{\beta_{\text {jol }} \underline{X}_{j k}^{u}-o_{k}^{l}}{\beta_{\text {jol }} \sum_{j=1}^{n_{j}} \underline{f}_{k}^{\mu}+\left(1-\beta_{\text {jol }}\right) \sum_{j=1}^{n_{j}} \underline{f}_{k}^{v}} \frac{\partial \underline{f}_{j}^{u}}{\partial \overline{\bar{c}}_{i j}^{o}}+\frac{\left(1-\beta_{\text {jol }}\right) \underline{\chi}_{j k}^{v}-o_{k}^{l}}{\beta_{\text {jol }} \sum_{j=1}^{n_{j}} \underline{f}_{k}^{\mu}+\left(1-\beta_{\text {jol }}\right) \sum_{j=1}^{n_{j}} \underline{f}_{k}^{v}} \frac{\partial \underline{f}_{j}^{v}}{\partial \overline{\bar{c}}_{i j}^{o}} \\
+\frac{\beta_{\text {jor }} \bar{\chi}_{j k}^{u}-o_{k}^{r}}{\beta_{\text {jor }} \sum_{j=1}^{n_{j}} \bar{f}_{k}^{\mu}+\left(1-\beta_{\text {jor }}\right) \sum_{j=1}^{n_{j}} \bar{f}_{k}^{v}} \frac{\partial \bar{f}_{j}^{u}}{\partial \overline{\bar{c}}_{i j}^{o}}+\frac{\left(1-\beta_{\text {jor }}\right) \bar{\chi}_{j k}^{v}-o_{k}^{r}}{\beta_{\text {jor }} \sum_{j=1}^{n_{j}} \bar{f}_{k}^{\mu}+\left(1-\beta_{\text {jor }}\right) \sum_{j=1}^{n_{j}} \bar{f}_{k}^{v}} \frac{\partial \bar{f}_{j}^{v}}{\partial \overline{\bar{c}}_{i j}^{o}}
\end{array}\right), \\
& \frac{\partial s(t) \dot{s}(t)}{\partial \underline{\underline{c}}_{i j}^{o}}=\sum_{k=1}^{n_{k}}\left(\frac{\partial s(t) \dot{s}(t)}{\partial \hat{u}_{\text {TT2FIBELN }_{k}}} \frac{\partial \hat{u}_{\text {IT2FIBELN }_{k}}}{\partial o_{k}}\left(\frac{\partial o_{k}}{\partial o_{k}^{l}} \frac{\partial o_{k}^{l}}{\partial f_{j}^{l}} \frac{\partial f_{j}^{l}}{\partial \underline{\hat{c}}_{i j}^{o}}+\frac{\partial o_{k}}{\partial o_{k}^{r}} \frac{\partial o_{k}^{r}}{\partial f_{j}^{r}} \frac{\partial f_{j}^{r}}{\partial \underline{\underline{c}}_{i j}^{o}}\right)\right)
\end{aligned}
$$

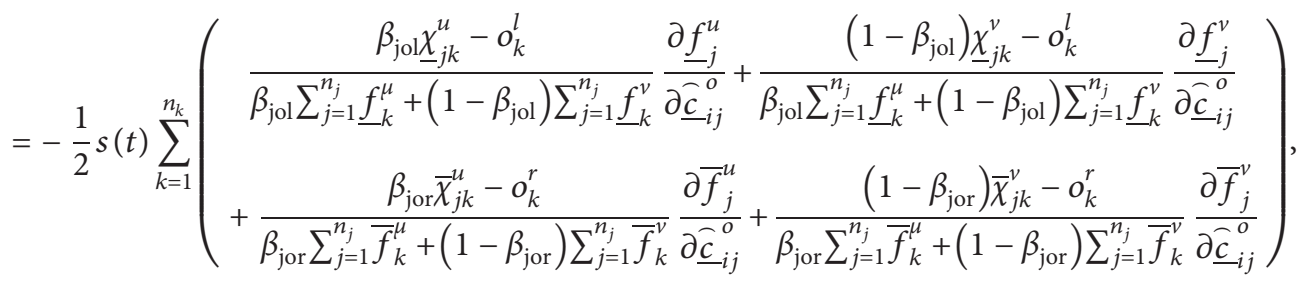

$$
\begin{aligned}
& \frac{\partial \underline{f}_{j}^{u}}{\partial \hat{\sigma}_{i j}^{a}}=\frac{\partial \underline{f}_{j}^{u}}{\partial \underline{\mu}_{i j}^{u}} \frac{\partial \underline{\mu}_{i j}^{u}}{\partial \hat{\sigma}_{i j}^{a}}=\underline{f}_{j}^{u} \frac{\left(I_{i}-\underline{\hat{c}}_{i j}^{a}\right)^{2}}{\left(\hat{\sigma}_{i j}^{a}\right)^{3}}, \\
& \frac{\partial \bar{f}_{j}^{u}}{\partial \hat{\sigma}_{i j}^{a}}=\frac{\partial \bar{f}_{j}^{u}}{\partial \bar{\mu}_{i j}^{u}} \frac{\partial \bar{\mu}_{i j}^{u}}{\partial \hat{\sigma}_{i j}^{a}}=\bar{f}_{j}^{u} \frac{\left(I_{i}-\hat{c}_{i j}^{a}\right)^{2}}{\left(\hat{\sigma}_{i j}^{a}\right)^{3}} \\
& \frac{\partial \underline{f}_{j}^{v}}{\partial \hat{\sigma}_{i j}^{a}}=\frac{\partial \underline{f}_{j}^{v}}{\partial \underline{v}_{i j}^{v}} \frac{\partial \underline{v}_{i j}^{v}}{\partial \hat{\sigma}_{i j}^{a}}=\underline{f}_{j}^{v} \frac{\left(I_{i}-\hat{\underline{c}}_{i j}^{a}\right)^{2}}{\left(\hat{\sigma}_{i j}^{a}\right)^{3}}, \\
& \frac{\partial \bar{f}_{j}^{v}}{\partial \hat{\sigma}_{i j}^{a}}=\frac{\partial \bar{f}_{j}^{v}}{\partial \bar{v}_{i j}^{v}} \frac{\partial \bar{v}_{i j}^{v}}{\partial \hat{\sigma}_{i j}^{a}}=\bar{f}_{j}^{v} \frac{\left(I_{i}-\hat{\underline{c}}_{i j}^{a}\right)^{2}}{\left(\hat{\sigma}_{i j}^{a}\right)^{3}}
\end{aligned}
$$




$$
\begin{aligned}
& \frac{\partial \underline{f}_{j}^{u}}{\partial \widehat{\sigma}_{i j}^{o}}=\frac{\partial \underline{f}_{j}^{u}}{\partial \underline{\mu}_{i j}^{u}} \frac{\partial \underline{\mu}_{i j}^{u}}{\partial \widehat{\sigma}_{i j}^{p}}=\underline{f}_{j}^{u} \frac{\left(I_{i}-\underline{\widehat{c}}_{i j}^{o}\right)^{2}}{\left(\widehat{\sigma}_{i j}^{o}\right)^{3}}, \\
& \frac{\partial \bar{f}_{j}^{u}}{\partial \widehat{\sigma}_{i j}^{o}}=\frac{\partial \bar{f}_{j}^{u}}{\partial \bar{\mu}_{i j}^{u}} \frac{\partial \bar{\mu}_{i j}^{u}}{\partial \hat{\sigma}_{i j}^{o}}=\bar{f}_{j}^{u} \frac{\left(I_{i}-\overline{\bar{c}}_{i j}^{o}\right)^{2}}{\left(\hat{\sigma}_{i j}^{o}\right)^{3}}, \\
& \frac{\partial \underline{f}_{j}^{v}}{\partial \widehat{\sigma}_{i j}^{o}}=\frac{\partial \underline{f}_{j}^{v}}{\partial \underline{v}_{i j}^{v}} \frac{\partial \underline{v}_{i j}^{v}}{\partial \widehat{\sigma}_{i j}^{o}}=\underline{f}_{j}^{v} \frac{\left(I_{i}-\underline{\hat{c}}_{i j}^{o}\right)^{2}}{\left(\widehat{\sigma}_{i j}^{o}\right)^{3}}, \\
& \frac{\partial \bar{f}_{j}^{v}}{\partial \bar{\sigma}_{i j}^{o}}=\frac{\partial \bar{f}_{j}^{v}}{\partial \bar{v}_{i j}^{v}} \frac{\partial \bar{v}_{i j}^{v}}{\partial \bar{\sigma}_{i j}^{o}}=\bar{f}_{j}^{v} \frac{\left(I_{i}-\overline{\bar{c}}_{i j}^{o}\right)^{2}}{\left(\bar{\sigma}_{i j}^{o}\right)^{3}}, \\
& \frac{\partial \underline{f}_{j}^{u}}{\partial \underline{\underline{c}}_{i j}^{a}}=\frac{\partial \underline{f}_{j}^{u}}{\partial \underline{\mu}_{i j}^{u}} \frac{\partial \underline{\mu}_{i j}^{u}}{\partial \underline{\underline{c}}_{i j}^{a}}=\left(1-\underline{\pi}_{c, i k}\right) \underline{f}_{j}^{u} \frac{I_{i}-\underline{\widehat{c}}_{i j}^{a}}{\left(\hat{\sigma}_{i j}^{a}\right)^{2}}, \\
& \frac{\partial \bar{f}_{j}^{u}}{\partial \underline{\underline{c}}_{i j}^{a}}=\frac{\partial \bar{f}_{j}^{u}}{\partial \bar{\mu}_{i j}^{u}} \frac{\partial \bar{\mu}_{i j}^{u}}{\partial \underline{\underline{c}}_{i j}^{a}}=\left(1-\bar{\pi}_{c, i k}\right) \bar{f}_{j}^{u} \frac{I_{i}-\underline{\hat{c}}_{i j}^{a}}{\left(\bar{\sigma}_{i j}^{a}\right)^{2}}, \\
& \frac{\partial \underline{f}_{j}^{v}}{\partial \underline{\widehat{c}}_{i j}^{a}}=\frac{\partial \underline{f}_{j}^{v}}{\partial \underline{v}_{i j}^{v}} \frac{\partial \underline{v}_{i j}^{v}}{\partial \underline{\underline{c}}_{i j}^{a}}=-\left(1-\underline{\pi}_{c, i k}\right) \underline{f}_{j}^{v} \frac{I_{i}-\underline{\widehat{c}}_{i j}^{a}}{\left(\hat{\sigma}_{i j}^{a}\right)^{2}}, \\
& \frac{\partial \bar{f}_{j}^{v}}{\partial \underline{\hat{c}}_{i j}^{a}}=\frac{\partial \bar{f}_{j}^{v}}{\partial \bar{v}_{i j}^{v}} \frac{\partial \bar{v}_{i j}^{v}}{\partial \underline{\hat{c}}_{i j}^{a}}=-\left(1-\bar{\pi}_{c, i k}\right) \bar{f}_{j}^{v} \frac{I_{i}-\overline{\bar{c}}_{i j}^{a}}{\left(\bar{\sigma}_{i j}^{a}\right)^{2}}, \\
& \frac{\partial \underline{f}_{j}^{u}}{\partial \overline{\bar{c}}_{i j}^{a}}=\frac{\partial \underline{f}_{j}^{u}}{\partial \underline{\mu}_{i j}^{u}} \frac{\partial \underline{\mu}_{i j}^{u}}{\partial \overline{\bar{c}}_{i j}^{a}}=\left(1-\underline{\pi}_{c, i k}\right) \underline{f}_{j}^{u} \frac{I_{i}-\overline{\bar{c}}_{i j}^{a}}{\left(\hat{\sigma}_{i j}^{a}\right)^{2}}, \\
& \frac{\partial \bar{f}_{j}^{u}}{\partial \overline{\bar{c}}_{i j}^{a}}=\frac{\partial \bar{f}_{j}^{u}}{\partial \bar{\mu}_{i j}^{u}} \frac{\partial \bar{\mu}_{i j}^{u}}{\partial \overline{\bar{c}}_{i j}^{a}}=\left(1-\bar{\pi}_{c, i k}\right) \bar{f}_{j}^{u} \frac{I_{i}-\overline{\bar{c}}_{i j}^{a}}{\left(\widehat{\sigma}_{i j}^{a}\right)^{2}}, \\
& \frac{\partial \underline{f}_{j}^{v}}{\partial \overline{\bar{c}}_{i j}^{a}}=\frac{\partial \underline{f}_{j}^{v}}{\partial \underline{v}_{i j}^{v}} \frac{\partial \underline{v}_{i j}^{v}}{\partial \overline{\bar{c}}_{i j}^{a}}=-\left(1-\underline{\pi}_{c, i k}\right) \underline{f}_{j}^{v} \frac{I_{i}-\overline{\bar{c}}_{i j}^{a}}{\left(\bar{\sigma}_{i j}^{a}\right)^{2}}, \\
& \frac{\partial \bar{f}_{j}^{v}}{\partial \overline{\bar{c}}_{i j}^{a}}=\frac{\partial \bar{f}_{j}^{v}}{\partial \bar{v}_{i j}^{v}} \frac{\partial \bar{v}_{i j}^{v}}{\partial \overline{\bar{c}}_{i j}^{a}}=-\left(1-\bar{\pi}_{c, i k}\right) \bar{f}_{j}^{v} \frac{I_{i}-\overline{\bar{c}}_{i j}^{a}}{\left(\hat{\sigma}_{i j}^{a}\right)^{2}}, \\
& \frac{\partial s(t) \dot{s}(t)}{\partial \pi_{\mathrm{val}, j k}^{a}}=\frac{\partial s(t) \dot{s}(t)}{\partial \widehat{u}_{\mathrm{IT}_{2 \mathrm{FiBELN}}}} \frac{\partial \bar{u}_{\mathrm{IT}_{k} \mathrm{FiBELN}_{k}}}{\partial a_{k}} \frac{\partial a_{k}}{\partial a_{k}^{l}} \frac{\partial a_{k}^{l}}{\partial \pi_{\mathrm{val}, j k}^{a}}=-\frac{1}{2} s(t) \frac{\left(1-\beta_{\mathrm{jol}}\right)_{k}^{v}}{\beta_{\mathrm{jol}} \sum_{j=1}^{n_{j}} \underline{f}_{k}^{\mu}+\left(1-\beta_{\mathrm{jol}}\right) \sum_{j=1}^{n_{j}} \underline{f}_{k}^{v}} .
\end{aligned}
$$


By using the updating laws, IT2FIBELN can approach the optimal parameters, and the control system can achieve the desired control performance.

\section{Self-Organizing IT2IFBELNHC Robust Control for the Multiple Degree-of-Freedom Rehabilitation Robot}

The structure of the self-organizing type-2 intuition fuzzy brain learning network hybrid controller (IT2IFBELNHC) is illustrated in Figure 2, which includes three interconnected subsystems: one sliding surface generator, one IT2IFBELN controller, and one robust controller, which is a compensation for the uncertainty and residual error.

The objective of the hybrid controller is to match the system trajectory $x(t)$ with the desired reference one ${ }_{x d}(t)$; $x(t)=\left[\begin{array}{llll}x_{1}(t) & x_{2}(t) & \cdots & x_{\kappa}(t)\end{array}\right]^{T} \quad$ and $\quad \underline{x}(t)=$ $\left[\begin{array}{llll}x^{T}(t) & \dot{x}^{T}(t) & \cdots & x^{(n-1) T}(t)\end{array}\right]^{T}$ denote the output and the state vector of the control system, respectively. Then, the tracking error could be obtained as $e(t)={ }_{x d}(t)-x(t)$, and the trajectory error vector could be defined as

$$
\underline{e}(t)=\left[\begin{array}{llll}
e^{T}(t) & e^{T}(t) & \cdots & e^{(n-1) T}(t)
\end{array}\right]^{T} .
$$

The sliding surface referred in the control scheme is defined as

$$
\begin{aligned}
s(\underline{e}(t))= & e^{(n-1)}(t)+\varsigma_{1} e^{(n-1)}(t)+\cdots+\varsigma_{n-1} e^{(n-1)}(t) \\
& +\varsigma_{n} \int_{0}^{t} e(t) \mathrm{d}(t),
\end{aligned}
$$

where $s=\left[\begin{array}{llll}s_{1} & s_{2} & \cdots & s_{\kappa}\end{array}\right]^{T}, \varsigma_{i}=\operatorname{diag}\left(\begin{array}{llll}\varsigma_{i 1} & \varsigma_{i 2} & \cdots & \varsigma_{i \kappa}\end{array}\right)$, and the constant is $\varsigma_{i}$ which is designed to make sure of the satisfaction of the Horowitz characteristic polynomial and should be positive; then differentiating $s(\underline{e}(t))$, we can get

$$
s(t)=e^{(n)}(t)+\varsigma_{1} e^{(n-1)}(t)+\cdots+\varsigma_{n} e(t)=\stackrel{(n)}{e}(t)+\varsigma^{T} \underline{e}(t),
$$

where $\varsigma=\left[\begin{array}{llll}\varsigma_{n} & \varsigma_{n-1} & \cdots & \varsigma_{1}\end{array}\right]^{T}$ means the gain matrix. Then, we can get the optimal control law given as

$$
u^{*}(t)=u_{\mathrm{IT2IFBELNC}}^{*}(t)+\varepsilon(t),
$$

where $\varepsilon(t)$ means the approximation error, so the robust compensation is added here to deal with this error. The optimal controller $u^{*}(t)$ cannot be obtained directly, so the estimation controller $\widehat{u}_{\text {IT2IFBELNC }}$ is needed here to estimate the optimal controller $u_{\mathrm{IT} 2 \mathrm{IFBELNC}}^{*}$; then, the control law can be modified as

$$
\begin{aligned}
u(t) & =\widehat{u}_{\mathrm{IT2IFBELNC}}(t)+\widehat{u}_{r}(t), \\
u_{r}(t) & =\widehat{N}(t) \operatorname{sgn}(s(t)),
\end{aligned}
$$

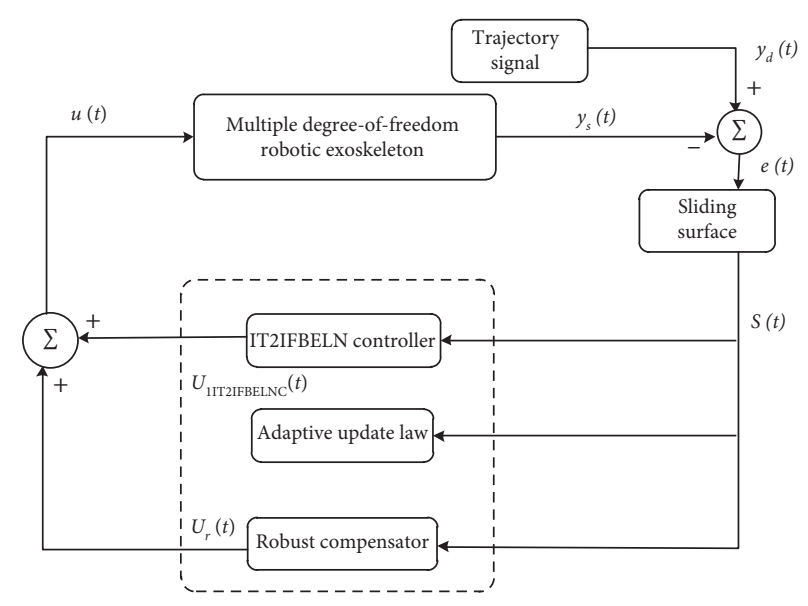

Figure 2: Adaptive control of IT2IFBELNHC for the robot.

where $\widehat{u}_{r}(t)$ denotes the robust controller and $\widehat{N}(t)$ represents the estimation value of uncertainty bound $N$, assuming that the approximation error can be bound as $0 \leq \varepsilon(0) \leq N$. The adaptive law for updating the bound is chosen as $\widehat{N}(t)=\eta_{r}|s(t)|$, where $\eta_{r}$ is the learning rate. Now, the robust controller could cover the approximation error by using this adaptive law. Then, the error equation can be obtained as

$$
s(\underline{e}(t))=e^{(n)}(t)+\varsigma^{T} \underline{e}(t)=\varepsilon(t)-u_{r}(t) .
$$

The Lyapunov function is defined as

$$
\begin{aligned}
V(s(t), \widetilde{D}(t)) & =\frac{1}{2} s^{2}(t)+\frac{\widetilde{D}^{2}(t)}{2 \eta_{r}}, \\
\widetilde{D}(t) & =D-\widehat{D}(t) .
\end{aligned}
$$

Then, taking the derivative of the above equation, we can get

$$
\dot{V}(s(t), \widetilde{D}(t))=s(t) \dot{\mathcal{s}}(t)+\frac{\widetilde{D}(t) \dot{\tilde{D}}(t)}{2 \eta_{r}}=-|s(t)(D-|\varepsilon(t)|)| \leq 0 .
$$

From the above equation, we can see that $V(s(t), \widetilde{D}(t)) \leq V_{1}(s(0), \widetilde{D}(0)), V(s(0), \widetilde{D}(0))$ is bounded, and $V(s(t), \widetilde{D}(t))$ is bounded and increasing. Meanwhile, we define one function $\Phi=(D-\varepsilon(t)) s \leq(D-|\varepsilon(t)|)|s|$ $\leq \dot{V}(s(t), \widetilde{D}(t))$ and then integrate this function, so the following expression is obtained:

$$
\int_{0}^{t} \Phi(\tau) \mathrm{d} \tau \leq V(s(0), \widetilde{D}(0))-\dot{V}(s(t), \widetilde{D}(t))<\infty .
$$




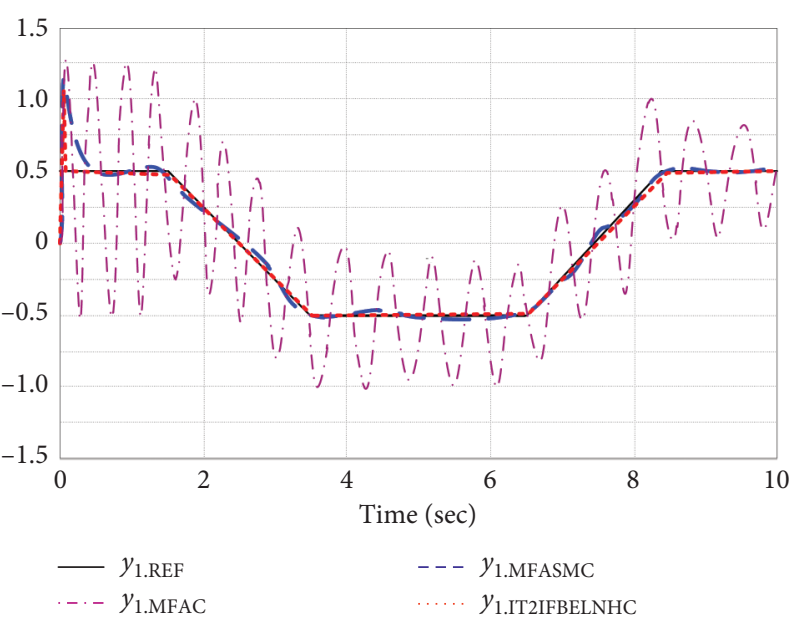

(a)

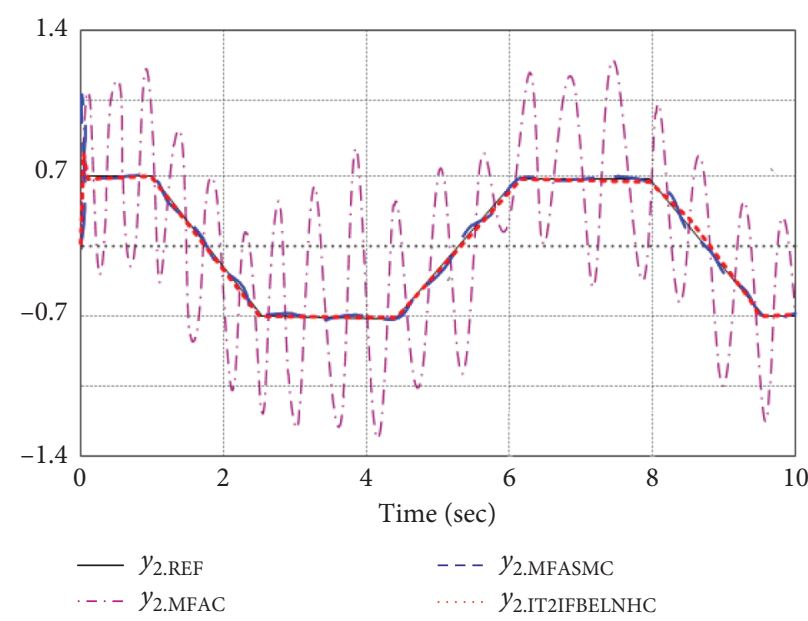

(b)

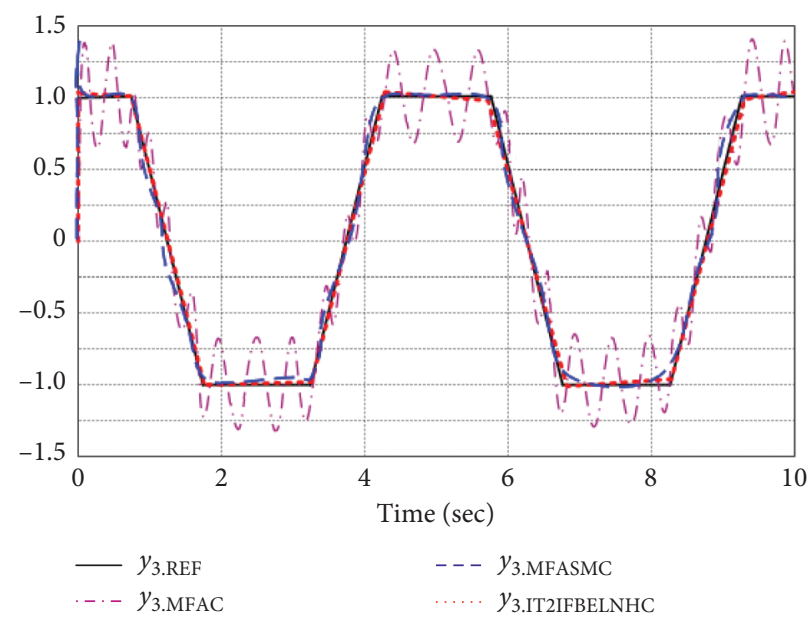

(c)

FIGURE 3: Tracking performance of (a) $y_{1}(k)$, (b) $y_{2}(k)$, and (c) $y_{3}(k)$ under three control methods: MFAC/MFSMAC/IT2IFBELNHC.

According to the Barbarella lemma, it can be seen that this control system based on the proposed method can achieve robust control performance.

\section{Simulation and Results}

The mechanical structure of this rehabilitation robot has been shown in reference $[14,25]$. In this simulation experiment, the wrist two joints are kept unchanged, and the other three joints will be controlled by the different controllers, which include the interval type- 2 intuition fuzzy brain learning network hybrid controller (IT2IFBELNHC), model-free adaptive sliding mode control (MFASMC) [25], and model-free adaptive control (MFAC) [16]. The simulation results will demonstrate the superiority of the proposed algorithm, and the simulation time is $10 \mathrm{~s}$.

5.1. Controlling Three-Joint Robot. The desired angle velocities $[1,2]$ of the robot have been set as follows:

$$
\begin{aligned}
& y_{d 1}= \begin{cases}-0.5, & y_{d 1}<-0.5, \\
\frac{2 \pi}{10} \cos \left(\frac{2 \pi}{10} t\right), & -0.5 \leq y_{d 1} \leq 0.5, \\
0.5, & y_{d 1}>0.5,\end{cases} \\
& y_{d 2}= \begin{cases}-0.7, & y_{d 2}<-0.7, \\
\frac{2 \pi}{7} \cos \left(\frac{2 \pi}{7} t\right), & -0.7 \leq y_{d 2} \leq 0.7, \\
0.7, & y_{d 2}>0.7,\end{cases} \\
& y_{d 3}= \begin{cases}-1.0, & y_{d 3}<-1.0, \\
\frac{2 \pi}{5} \cos \left(\frac{2 \pi}{5} t\right), & -1.0 \leq y_{d 3} \leq 1.0, \\
1.0, & y_{d 3}>1.0 .\end{cases}
\end{aligned}
$$




\subsection{Performance of Model-Free Adaptive Control.} Figure 3 demonstrates the simulation results used by the model-free adaptive control scheme, where Figures 3(a)3 (c) show the performances of outputs $y_{1 \text { MFAC }}, y_{2 . \mathrm{MFAC}}$, and $y_{3 \text { MFAC. }}$. Figure 4 shows the control inputs of $u_{1 \text { MFAC }}, u_{2 . \mathrm{MFAC}}$, and $u_{3 \text {.MFAC }}$, respectively. From the results, we can see that control inputs have some fluctuations.

5.3. Performance of Model-Free Adaptive Sliding Mode Control. Figures 3 and 5 show the control performance using the model-free adaptive sliding mode control method, where Figures 3(a)-3(c) show control performances of outputs of $y_{1 \text {.MFASMC }}, y_{2 . M F A C S M}$, and $y_{3 . M F A S M C}$, from which we can see that the convergence of the method is slow. Figure 5 shows control inputs of $u_{1 \text { MFASMC }}, u_{2 \text { MFASMC, }}$, and $u_{3 \text { MFASMC }}$, respectively, from which we can see that the control performance of the MFASMC on the rehabilitation robot is tracking well, but has a big fluctuation at first.

5.4. Performance of Model-Free Adaptive Sliding Mode Combined with Neural Network Control. This part shows the control performance of the model-free sliding mode combined with the neural network control method, and the initial values are chosen the same as in reference [29] and shown as

$$
\begin{aligned}
y(k-1) & =[0,0,0]^{T}, \\
y(k-2) & =[0,0,0]^{T}, \\
u(k-1) & =[0,0,0]^{T}, \\
u(k-2) & =[0,0,0]^{T}, \\
\varphi_{1} & =\operatorname{diag}[1,1,1], \\
\varphi_{2} & =\operatorname{diag}[1,1,1] .
\end{aligned}
$$

The controller parameters are $\eta=\rho=\lambda=\mu=1$, $q=600$, and $\Gamma=\operatorname{diag}([6,6,1])$.

Figures 3 and 6 also show the control performance of the model-free adaptive sliding mode combined with the neural network method, where Figures 3(a)-3(c) show the performances of outputs, from which we can see that the tracking results perform quite satisfactory. Figure 6 presents the control inputs, from which we also can see that control inputs are quite stable.

5.5. Comparison Analysis. In this part, the comparisons have been done to demonstrate the control performance of the proposed method, and for this, the root mean square (RMS) values of errors are created by the three kinds of controllers, and the results are shown in Table 1, respectively. From the comparison results of the RMS values, we can conclude that the convergence performance and speed of the proposed method are better than two other controllers although the proposed controller has a little overshoot at the first time.

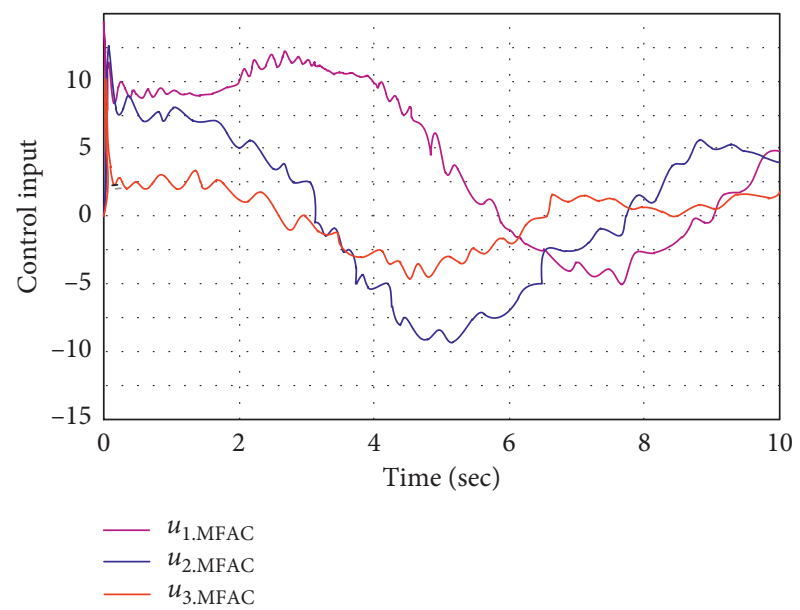

FIGURE 4: Control inputs of $u_{1}(k), u_{2}(k)$, and $u_{3}(k)$ under MFAC.

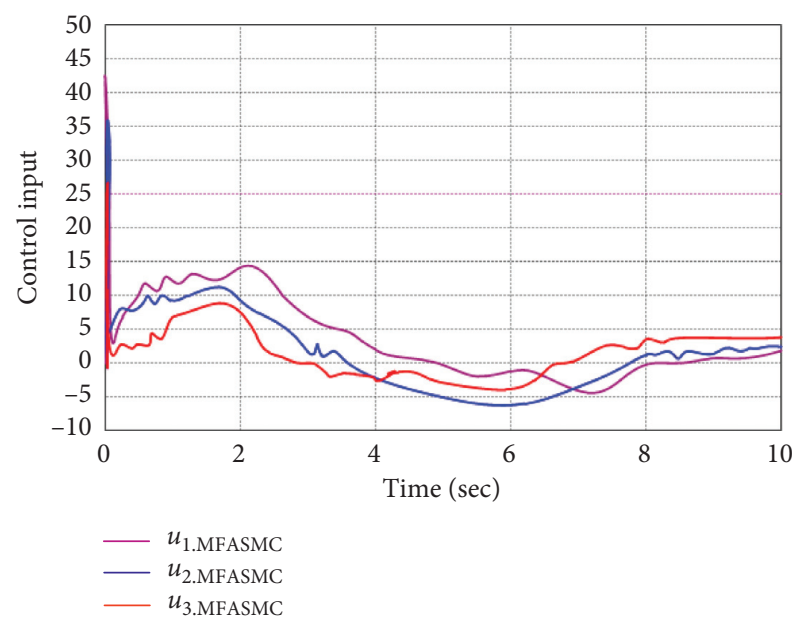

FIGURE 5: Control inputs of $u_{1}(k), u_{2}(k)$, and $u_{3}(k)$ under MFASMC.

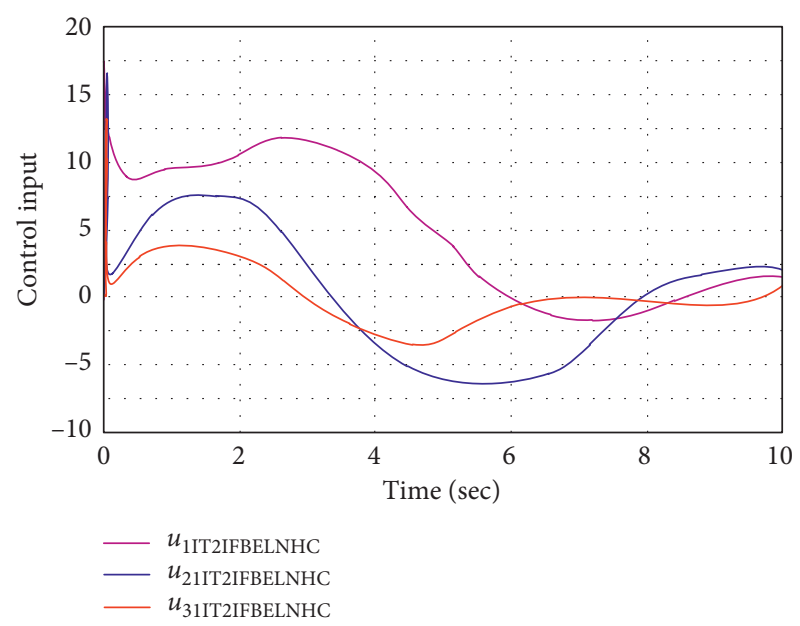

FIgURE 6: Control inputs of $u_{1}(k), u_{2}(k)$, and $u 3(k)$ under IT2IFBELNHC. 
TABLE 1: Comparison of RMS values.

\begin{tabular}{lcccccc}
\hline \multicolumn{2}{c}{ RMS of error } & $(0,2]$ & $(2,4]$ & $(4,6]$ & $(6,8]$ & $(8,10]$ \\
\hline \multirow{3}{*}{ MFAC } & $e 1$ & 0.0206 & 0.0022 & 0.0032 & 0.0033 & 0.0028 \\
& $e 2$ & 0.0261 & 0.0035 & 0.0021 & 0.0029 & 0.0019 \\
& $e 3$ & 0.0138 & 0.0019 & 0.0019 & 0.0017 & 0.0018 \\
\hline \multirow{3}{*}{ MFASMC } & $e 1$ & 0.0198 & 0.0019 & 0.0029 & 0.0023 & 0.0026 \\
& $e 2$ & 0.0168 & 0.0028 & 0.0019 & 0.0018 & 0.0016 \\
\multirow{3}{*}{ IT2IFBELNFC } & $e 3$ & 0.0118 & 0.0015 & 0.0016 & 0.0015 & 0.0013 \\
& $e 1$ & 0.0176 & 0.0015 & 0.0018 & 0.0012 & 0.0012 \\
& $e 2$ & 0.0131 & 0.0022 & 0.0016 & 0.0014 & 0.0012 \\
& $e 3$ & 0.0124 & 0.0013 & 0.0013 & 0.0011 & 0.0011 \\
\hline
\end{tabular}

\section{Conclusions}

In this paper, one novel fuzzy neural network which integrates the self-organizing type- 2 intuition fuzzy sets and brain emotional learning network has been proposed. The rules of the proposed network can be self-constructed; the parameters of the network can be tuned based on the gradient descent method, and also the stability of the updating laws is guaranteed by the convergence analysis using Lyapunov theorem. Finally, the numerical simulation results demonstrate that the multiple degree-of-freedom rehabilitation robot using the self-organizing type-2 intuition fuzzy brain emotional learning network controller led to more precise position tracking and more favorable stability in comparison with other methods. In future work, we will concentrate on reducing time of training networks.

\section{Data Availability}

The data used to support the findings of this study are available from the corresponding author upon request.

\section{Conflicts of Interest}

The authors declare that they have no conflicts of interest.

\section{Acknowledgments}

The authors are grateful for the support of the http://doi.org/ 10.13039/501100001809 National Science Foundations of China (nos. 61502211, 61572242, and 61702234), Key Laboratory of Computational Intelligence and Chinese Information Processing, and Key Laboratory of Intelligent Information Processing in Shanxi Province (CICIP2020004).

\section{References}

[1] N. N. Gheidari, "Effects of robot-assisted therapy on stroke rehabilitation in upper limbs: systematic review and metaanalysis of the literature," Journal of Rehabilication of Research and Develop, vol. 49, pp. 479-495, 2012.

[2] H.-B. Kang and J.-H. Wang, "Adaptive robust control of 5 DOF upper-limb exoskeleton robot," International Journal of Control, Automation and Systems, vol. 13, no. 3, pp. 733-741, 2015.

[3] Q. Li, D. Wang, Z. Du, and L. Sun, "A novel rehabilitation system for upper limbs," in Proceedings of the 27th Annual International Conference of the Engineering in Medicine and
Biology Society, pp. 6840-6843, IEEE-EMBS, Shanghai, China, September 2005.

[4] H. S. Lo and S. Q. Xie, "Exoskeleton robots for upper-limb rehabilitation: state of the art and future prospects," Medical Engineering \& Physics, vol. 34, no. 3, pp. 261-268, 2012.

[5] C. Carignan, J. Tang, and S. Roderick, "Development of an exoskeleton haptic interface for virtual task training," in Proceedings of the IEEE/RSJ International Conference on Intelligent Robots and Systems, pp. 3697-3702, IROS-IEEE, St. Louis, MO, USA, October 2009.

[6] R. A. R. C. Gopura, K. Kiguchi, and Y. L. Sueful, "7DOF upper-limb exoskeleton robot with muscle-model-oriented EMG-based control," in Proceedings of the IEEE/RSJ International Conference on Intelligent Robots and Systems, pp. 1126-1131, IROS-IEEE, St. Louis, MO, USA, October 2009.

[7] Y. Liu, B. Niu, F. Chu, and Y. Liu, "Adaptive fuzzy outputfeedback tracking control for a class of switched stochastic nonlinear time-delay systems," Circuits, Systems, and Signal Processing, vol. 35, no. 8, pp. 2762-2788, 2016.

[8] T. Nef, M. Guidali, and R. Riener, "ARMin-arm therapy exoskeleton with an ergonomic shoulder actuation," Application of Bionics Biomechanics, vol. 6, pp. 127-142, 2016.

[9] A. H. Stienen, E. E. Hekman, F. C. Van der Helm et al., "Dampace: dynamic force-coordination trainer for the upper extremities," in Proceedings of the Rehabilitation Robotics, 2007. ICORR 2007, IEEE 10th International Conference, pp. 820-826, IEEE, Noordwijk, The Netherlands, June 2017.

[10] R. Vertechy, A. Frisoli, A. Dettori, and M. Solazzi Bergamasc, "Development of a new exoskeleton for upper limb rehabilitation," in Proceedings of the IEEE International Conference on Rehabilitation Robotics, 2009, ICORR 2009, pp. 188-193, IEEE, Kyoto, Japan, June 2009.

[11] H. Liu, Y. Pan, J. Cao, Y. Zhou, and H. Wang, "Positivity and stability analysis for fractional-order delayed systems: a T-S fuzzy model approach," IEEE Transactions on Fuzzy Systems, 2020.

[12] H. Liu, Y. Pan, J. Cao, H. Wang, and Y. Zhou, "Adaptive neural network backstepping control of fractional-order nonlinear systems with actuator faults," IEEE Transactions on Neural Networks and Learning Systems, vol. 31, no. 12, p. 5166, 2020.

[13] H. Liu, Y. Pan, and J. Cao, "Composite learning adaptive dynamic surface control of fractional-order nonlinear systems," IEEE Transactions on Cybernetics, vol. 50, no. 6, pp. 2557-2567, 2019.

[14] H. Liu, Y. Pan, S. Li, and Y. Chen, "Adaptive fuzzy backstepping control of fractional-order nonlinear systems," IEEE Transactions on Systems, Man, and Cybernetics: Systems, vol. 47, no. 8, pp. 2209-2217, 2017.

[15] S. Yin, S. X. Ding, X. Xie, and H. Luo, "A review on basic datadriven approaches for industrial process monitoring," IEEE Transactions on Industrial Electronics, vol. 61, no. 11, pp. 6418-6428, 2014.

[16] Z. S. Hou and S. T. Jin, Model-free Adptive Control: Theory and Applications, Science Press, Beijing, China, 2013.

[17] S. Formentin, P. De Filippi, M. Corno, M. Tanelli, and S. M. Savaresi, "Data-driven design of braking control systems," IEEE Transactions on Control Systems Technology, vol. 21, no. 1, pp. 186-193, 2013.

[18] G. Wang and Z. Huang, "Data-driven fault-tolerant control design for wind turbines with robust residual generator," IET Control Theory \& Applications, vol. 9, no. 7, pp. 1173-1179, 2015. 
[19] D. Xu, B. Jiang, and P. Shi, "A novel model-free adaptive control design for multivariable industrial processes," IEEE Transactions on Industrial Electronics, vol. 61, no. 11, pp. 6391-6398, 2015.

[20] R. M. Cao, H. X. Zhou, and Z. S. Hou, "Data-driven modelfree adaptive precision control for linear servo system," Control Theory and Applications, vol. 29, no. 3, pp. 310-316, 2012.

[21] X. J. Chen, D. Li, B. X. Yang, and P. X. Wang, "Model-free adaptive sliding mode robust control with neural network estimator for the multi-degree-of-freedom robotic exoskeleton," Complexity, vol. 2020, Article ID 8327456, 2020.

[22] J. LeDoux, "Emotion and the limbic system concept," Concepts Neuroscience, vol. 2, pp. 169-199, 1991.

[23] J. E. LeDoux, "Emotion circuits in the brain," Annual Review of Neuroscience, vol. 23, no. 1, pp. 155-184, 2000.

[24] C. M. Lin and C. C. Chung, "Fuzzy brain emotional learning control system design for nonlinear systems," International Journal of Fuzzy System, vol. 17, no. 2, pp. 119-128, 2015.

[25] X. Wang, X. Li, J. Wang, X. Fang, and X. Zhu, "Data-driven model-free adaptive sliding mode control for the multi degree-of-freedom robotic exoskeleton," Information Sciences, vol. 327, pp. 246-257, 2016.

[26] J. Ayubi, A. Omidi, S. M. Barakati, and P. Ayubi, "Short term load forecasting based on brain emotional predictor," in Proceedings of the 20th Iranian Electrical Power Distribution Conference EPDC, pp. 37-41, Zahedan, Iran, April 2015.

[27] M. Jafari, R. Fehr, L. R. G. Carrillo, and H. Xu, "Brain emotional learning-based intelligent tracking control for unmanned aircraft systems with uncertain system dynamics and disturbance," in Proceedings of the International Conference on Unmanned Aircraft Systems ICUAS, pp. 1470-1475, Miami, FL USA, June 2017.

[28] M. Jafari and S. B. Shuraki, "Speed control of a digital servo system using brain emotional learning based intelligent controller," in Proceedings of the International Power Electronics, Drive Systems and Technologies Conference PEDSTC, pp. 311-314, Mashhad, Iran, February 2017.

[29] S. Khorashadizadeh and M. Mahdian, "Voltage tracking control of DC-DC boost converter using brain emotional learning," in Proceedings of the International Conference on Computational Intelligence and Applications ICCIA, pp. 268-272, Qazvin, Iran, January 2016.

[30] E. Lotfi, "Mathematical modeling of emotional brain for classification problems," in Proceedings of the Indian Academy of Management IAM, pp. 60-71, London, UK, November 2013.

[31] E. Lotfi and M.-R. Akbarzadeh-T, “Adaptive brain emotional decayed learning for online prediction of geomagnetic activity indices," Neurocomputing, vol. 126, pp. 188-196, 2014.

[32] M. A. Sharbafi, C. Lucas, and R. Daneshvar, "Motion control of omni-directional three-wheel robots by brain-emotionallearning-based intelligent controller," IEEE Transactions on Systems, Man, and Cybernetics, Part C (Applications and Reviews), vol. 40, no. 6, pp. 630-638, 2010.

[33] C. F. Hsu, C. T. Su, and T. T. Lee, "Chaos synchronization using brain-emotional learning-based fuzzy control," in Proceedings of the Scandinavian Conference on Information Systems, pp. 811-816, Odder, Denmark, August 2016.

[34] H. S. Milad, U. Farooq, M. E. El-Hawary, and M. U. Asad, "Neo-fuzzy integrated adaptive decayed brain emotional learning network for online time series prediction," IEEE Access, vol. 5, pp. 1037-1049, 2015.
[35] C.-F. Hsu and T.-T. Lee, "Emotional fuzzy sliding-mode control for unknown nonlinear systems," International Journal of Fuzzy Systems, vol. 19, no. 3, pp. 942-953, 2017.

[36] W. B. Fang, F. Chao, C. M. Lin, and L. Z. Yang, "An improved fuzzy brain emotional learning model network controller for humanoid robots," Frontiers in Neurorobotics, vol. 13, no. 2, pp. 1-16, 2019.

[37] J. Zhao, C. M. Lin, and F. Chao, "Wavelet fuzzy brain emotional learning control system design for MIMO uncertain nonlinear systems," Frontiers in Neurorobotics, vol. 12, pp. 1-17, 2019.

[38] F. Chao, D. J. Zhou, C. M. Lin, and L. Z. Yang, "Type-2 fuzzy hybrid controller network for robotic systems," IEEE Transactions on Cybernetics, vol. 50, no. 8, pp. 1-15, 2019.

[39] I. Eyoh, R. John, and G. De Maere, "Interval type-2 a-intuitionistic fuzzy logic for regression problems," IEEE Transactions on Fuzzy Systems, vol. 26, no. 4, pp. 2396-2408, 2018.

[40] I. Eyoh, R. John, and M. G. DE, "Extended kalman filter-based learning of interval type-2 intuition fuzzy logic system," in Proceedings of the Systems, Man, and Cybernetics (SMC), 2017 IEEE International Conference, pp. 728-773, IEEE, Banff, Canada, October 2017.

[41] T.-L. Le, C.-M. Lin, and T.-T. Huynh, "Self-evolving type-2 fuzzy brain emotional learning control design for chaotic systems using PSO," Applied Soft Computing, vol. 73, pp. 418-433, 2018. 